\title{
Fecal incontinence: major problem or incidental finding?
}

\author{
J. M. Devesa ${ }^{1}$
}

Received: 30 November 2015/Accepted: 30 November 2015/Published online: 28 December 2015

(C) Springer-Verlag Italia Srl 2015

It has been widely demonstrated that any degree of fecal incontinence (FI), even isolated and occasional leakage of gas, can seriously impair quality of life (QOL). The prevalence of FI ranges from $2.2 \%$ up to more than $50 \%$ in women with urinary incontinence or pelvic organ prolapse [1], increasing with advancing age in both sexes. According to these figures, in a Western population of 500,000 inhabitants, one would expect to find a minimum of 11,000 persons and a maximum of many thousands suffering from FI. Therefore, FI is a big problem. However, if one considers the number of those people who seek specialized care, and the number of patients finally treated, FI is an anecdote.

For instance, in the Ramon y Cajal Hospital, Madrid, a national reference center for FI currently serving a population of around 600,000 inhabitants, only about 75 patients, $0.6 \%$ of a minimum of 13,200 patients with this condition, are treated annually and, of those, less than half will achieve satisfactory long-term results. These numbers are very frustrating from both the social and medical aspects.

But why does this happen? There are some well-known reasons. As Paka et al. [2] state in their article, this symptom is underreported by patients, and patients are not routinely questioned about FI by general practitioners (GP) or geriatricians, who may be unaware of current treatments, which, in turn, are usually disappointing or very expensive. It is beyond the scope of this editorial to delve into the technical aspects of treatment; this would be a topic for another editorial. Instead, I want to focus on the need to

\footnotetext{
J. M. Devesa

colonrecto@ ruberinternacional.es

1 University Hospital Ramón y Cajal, Madrid, Spain
}

find a simple, not time-consuming, scoring system for assessing this disabling condition. This is the first essential step for recruiting patients from health centers and nursing homes.

Currently, three validated methods are more frequently used to score FI: the Jorge-Wexner (Cleveland Clinic) score: five items rated on a five-point scale; the Vaizey (St. Mark's Hospital) score: seven items, four rated on a fivepoint scale and three rated on a two- point scale; and the Fecal Incontinence Severity Index (FISI): four items rated on a six-point scale. The validity of these methods is beyond doubt and, up to now, they have been essential for determining the severity of FI, assessing periodically the response to treatment, and comparing the results of different therapeutic options. However, although one of them (Vaizey) also reports lifestyle alterations and qualitative aspects of FI, the final score is given by a single number that does not represent either the same degree of dysfunction or the different degree to which a given symptom affects a person's life. To overcome this, it would be necessary to measure each of the different qualitative aspects of FI quantitatively with independent scores, which is too complicated for routine use in clinical practice. Moreover, although none of these methods is difficult to use, the patient often has doubts or does not have episodes of diarrhea in the limited time, or uses the pad only for fear of leakage rather than for actual leakage, or even for urinary incontinence, which results in an imprecise score. In any case, it is highly unlikely that GPs or geriatricians routinely use any of these methods as an initial tool to assess FI.

For patients with FI, the true importance of incontinence lies in the way it affects their life; therefore, it is also necessary to know its impact on QOL, with the artificial number of any scoring system reflecting the qualitative 
states as well as possible. The impact on QOL is usually measured by the Fecal Incontinence Quality of Life (FIQOL) scale, (1 general question about health plus 26 items divided into 4 subscales: lifestyle, coping, depression, embarrassment) and the World Health Organization Quality of Life (WHOQOL) scale, (26 items divided into 4 domains: physical health, psychological, social, and environment). Recently, Reynolds et al. [3] introduced a system already used in numerous studies, the Disgust SensitivityRevised (DS-R) scale (27 items, 3 subscales: animal-reminder, contamination, and core disgust), to assess prospectively whether dispositional sensitivity to disgust predicts the QOL in patients with FI beyond the severity of incontinence symptoms, and whether or not it moderates the relationship between the severity of incontinence symptoms and QOL. Filling out any of these questionnaires is time-consuming, and they must be adapted to each country and are not easy for the patient to complete. Hence, they are generally used to strengthen work with a view to publication.

In the literature, there is not much written about correlations between the severity of FI and its impact on QOL. Reynolds et al. [3] demonstrated an inverse association between FISI and FIQOL lifestyle and depression subscales, the same as between FISI and WHOQOL physical subscale, and a marginal, but not significant, association between higher DS-R and poorer WHOQOL in the psychological domains. However, they found that FISI and DS-R were not associated with one another, indicating they were measuring distinct concerns.

In a recent editorial, Evans [4] emphasizes the growing interest in what is called patient-reported outcome measures (PROM). He asserts that insight into the patients' experience is gaining increasing importance. However, one study demonstrated the heterogeneity of the questionnaires, with the majority of them only used once, and little evidence of consistency between domains and questions examined.

A test must be accurate, thus measuring what it is intended to measure; reproducible, meaning that the same measurement taken on repeated occasions will have the same result; and responsive, in order to be able to detect change. Visual analogue scales (VAS) accomplish this and are simple and valid methods of scoring functional symptoms. VAS has proved to be a reliable, convenient, and accurate measure of bother that is related to urinary incontinence [5].

In 2013, my colleagues and I [6] published a study aimed to determine the possible correlation between a VAS for FI and a VAS for QOL, and the respective agreement of VAS for FI and the Jorge-Wexner score, as well as each VAS with each of the four subscales of FIQOL. The study showed that a VAS for FI cannot replace the Jorge-Wexner score and a VAS for QOL cannot replace all the four subscales of FIQOL. Furthermore, the severity of FI and its impact on QOL, expressed in a VAS for FI and a VAS for QOL, only have a fair correlation, showing that they do not assess the same issues, which is also supported by the finding that VAS for FI only correlates significantly with FIQOL embarrassment. Rockwood et al. [7] studied the correlation between FISI and FIQOL subscales and demonstrated significant correlations in lifestyle, coping/behavior, and embarrassment. In our opinion, this is a relevant finding, because evaluation of FI with FISI alone would also include a valid evaluation of QOL, and thus QOL would not have to be tested separately. Using a similar approach, we also tried to see whether VAS for FI correlates with FIQOL subscales, but we found that the correlation was only significant with embarrassment and to a lesser extent with coping/behavior, similar to the findings in Rockwood's study [7], suggesting that those are the aspects of QOL that are of the greatest concern to patients with FI. Curiously, in both studies the depression/self-perception subscale was not significantly correlated with the severity of the symptom, whatever the reason might be, and this feature could be taken into account if further studies aimed at simplifying FIQOL evaluation are undertaken. In other words, this studied demonstrated that, from the patients' perspective, intensity of incontinence does not run parallel with the impact on QOL.

In this issue of the journal, Paka et al. [2] aim to determine the correlation between a total Vaizey score and a VAS for bother FI and the correlation between individual components of the Vaizey score and VAS, and they conclude that there is a fair, positive correlation between VAS for bother and Vaizey and that patients' bother from FI is strongly associated with its impact on lifestyle as quantified by individual Vaizey components.

Although the VAS study by Paka and the one by my colleagues and I show different results, either due to different methods or statistical analysis, we think that a further multicenter study intended to validate VAS for FI is mandatory. The advantages are multiple: The questionnaire can be understood by everyone; it is quicker than any other test, easy for GPs or geriatricians to check and retest as many times as necessary.

Something as simple as a VAS, with the participation of GPs and geriatricians, would be of paramount importance for a better understanding and useful handling of FI.

Independently, for the purpose of clinical research, the use of one validated and objective score that measures both the intensity of FI and the impact of FI on QOL is still essential. However, it seems necessary to have a single system that is unanimously accepted and used as a reference in all works and studies on this big problem. 


\section{Compliance with ethical standards}

Conflict of interest The author declares that he has no conflict of interest.

Ethical approval All procedures performed in studies involving human participants were in accordance with the ethical standards of the institutional and/or national research committee and with the 1964 Helsinki declaration and its later amendments or comparable ethical standards.

Informed consent Informed consent was obtained from all individual participants included in the study.

\section{References}

1. De Mello Portella P, Feldner PC Jr, da Cionceicao JC, Castro RA, Sartori MG, Girao MJ (2012) Prevalence of and quality of life related to anal incontinence in women with urinary incontinence and pelvic organ prolapsed. Eur J Obstet Gynecol Reprod Biol 160:228-231
2. Paka C, Atan IK, Dietz HP (2015) The bother of anal incontinence and St Mark's Incontinence Score. Tech Coloproctol. doi:10.1007/ s10151-015-1397-z

3. Reynolds LM, Bissett NS, Consedine S (2015) Predicting the patients who will struggle with anal incontinence: sensitivity to disgust matters. Colorectal Dis 17:73-80

4. Evans MD (2015) The need for standardized Patient Reported Outcome Measures (PROM) in colorectal surgery. Colorectal Dis 17:937

5. Lukacz ES, Lawrence JM, Burchette RJ, Luber KM, Nager CW, Buckwalter JG (2004) The use of Visual Analog Scale in urogynecologic research: a psychometric evaluation. Am J Obstet Gynecol 191:165-170

6. Devesa JM, Vicente R, Abraira V (2013) Visual Analogue Scales for grading faecal incontinence and quality of life: their relationship with the Jorge-Wexner score and Rockwood scale. Tech Coloproctol 17:67-71

7. Rockwood TH, Church JM, Flesman JW et al (1999) Patient and surgeon ranking of the severity of symptoms associated with fecal incontinence: the fecal incontinence severity index. Dis Colon Rectum 42:1525-1532 